\title{
Signs of a vector's adaptive choice: on the evasion of infectious hosts and parasite-induced mortality
}

\author{
Fardo Witsenburg, Franziska Schneider and Philippe Christe \\ F. Witsenburg (fardo.witsenburg@unil.ch), F. Schneider and P. Christe, Dept of Ecology and Evolution, Univ. of Lausanne, Biophore, \\ UNIL-Sorge, CH-1015 Lausanne, Switzerland.
}

\begin{abstract}
Laboratory and field experiments have demonstrated in many cases that malaria vectors do not feed randomly, but show important preferences either for infected or non-infected hosts. These preferences are likely in part shaped by the costs imposed by the parasites on both their vertebrate and dipteran hosts. However, the effect of changes in vector behaviour on actual parasite transmission remains a debated issue.

We used the natural associations between a malaria-like parasite Polychromophilus murinus, the bat fly Nycteribia kolenatii and a vertebrate host the Daubenton's bat Myotis daubentonii to test the vector's feeding preference based on the host's infection status using two different approaches: 1) controlled behavioural assays in the laboratory where bat flies could choose between a pair of hosts; 2) natural bat fly abundance data from wild-caught bats, serving as an approximation of realised feeding preference of the bat flies.

Hosts with the fewest infectious stages of the parasite were most attractive to the bat flies that did switch in the behavioural assay. In line with the hypothesis of costs imposed by parasites on their vectors, bat flies carrying parasites had higher mortality. However, in wild populations, bat flies were found feeding more based on the bat's body condition, rather than its infection level. Though the absolute frequency of host switches performed by the bat flies during the assays was low, in the context of potential parasite transmission they were extremely high.

The decreased survival of infected bat flies suggests that the preference for less infected hosts is an adaptive trait. Nonetheless, other ecological processes ultimately determine the vector's biting rate and thus transmission. Inherent vector preferences therefore play only a marginal role in parasite transmission in the field. The ecological processes rather than preferences per se need to be identified for successful epidemiological predictions.
\end{abstract}

The success of a vector transmitted disease is, by its nature, not only defined by the ecology and behaviour of the host, but also by those of the vector. Given this, it is not surprising that an unprecedented amount of research is focused on mosquitoes and their role in malaria epidemiology (Lyimo and Ferguson 2009, Farajollahi et al. 2011). One key aspect of the vector's biology is its blood-feeding behaviour. Anthropophilic mosquitoes do not feed indiscriminately but show a preference for some blood donors over others, within a single host species (Knols et al. 1995, Liebman et al. 2014). Of particular interest for epidemiology is the feeding preference of the mosquito vector based on the host's level of infection. Such a preference is predicted to severely alter the dynamics and equilibrium level of infection of a given epidemiological system (Kingsolver 1987, Smith et al. 2014). This prediction, however, only holds if such a preference would actually result in differential biting rates of the more attractive hosts under natural conditions (Kingsolver 1987). Yet the relation between the vector's preference, its natural feeding behaviour and parasite transmission has still not been satisfyingly demonstrated (Cator et al. 2012).

Mosquito vectors are able to discriminate between hosts on the basis of the host infection status. Their feeding preference, sometimes referred to as 'host attractiveness', has been experimentally tested for numerous host species. In humans (Lacroix et al. 2005), rodents (Day et al. 1983, Ferguson and Read 2004), as well as in birds (Freier and Friedman 1976, Lalubin et al. 2012, Cornet et al. 2013) these choice experiments demonstrated either attraction to or avoidance of malaria-infected hosts, or no effect of the parasite. These contradictory results could be due to the choice of methodology (e.g. olfactory cues versus restricted body contact), the study system (e.g. natural versus unnatural host species; Tripet 2009), or generally to the geographic mosaic of coevolution that may lead to local adaptation. None of these studies, however, linked the preferences observed in the laboratory to the actual feeding rates of these vectors under natural conditions.

Measuring natural feeding rates of vectors on wild animals is methodologically challenging. Instead, approximations such as relative local vector abundances are used. Tomás et al. (2008) demonstrated that biting midges (Ceratopogonidae), the natural vectors of avian Haemoproteus haemosporidians, were more abundant in nests with malaria-treated blue tit females. Notably, the study could not demonstrate that the increased biting midge numbers were a direct consequence 
of the vector's preference, since the authors could not control for differential survival rates of the midges in the differently treated nests. As a result, a link between host preference and actual feeding patterns could not be established.

The aim of the present study was first to test under laboratory conditions the preference of the vector Nycteribia kolenatii for the host Myotis daubentonii based on the host's infection level with the haemosporidian parasite Polychromophilus murinus. A feeding preference for infected hosts might originate from many different processes, e.g. a host's lower anti-parasite behaviour or a parasite-based manipulation to increase its transmission success (Thomas et al. 2005, Cator et al. 2012). Alternatively, a feeding preference for uninfected hosts would be a strong indication that the parasite has a detrimental effect on the bat fly's fitness. To investigate this point, an experiment was performed to test the effects of $P$. murinus infection on the survival of bat flies. We furthermore tested the host fidelity of the bat fly by quantifying their host switching behaviour. Finally, results of host choice preference obtained in laboratory conditions were compared to the natural feeding rate of the vector. We used the relative abundances of $N$. kolenatii in a wild population of $M$. daubentonii as the approximation of the bat flies feeding rate. Since bat flies rarely venture off-host and take very frequent blood meals, their presence on a certain host should directly translate to their relative feeding rate on that host.

\section{Methods}

\section{Studied species}

Bat flies (Diptera: Nycteribiidae) are blood-sucking wingless ectoparasites which specialize on living in the fur of bats (Dick and Patterson 2006). Unlike most haematophagous Diptera, they do not engorge themselves, instead taking several blood meals per day, from once every hour up to every eight minutes (Marshall 1970, Overal 1980, Fritz 1983). Like all members of the Hippoboscoidea superfamily, they are viviparous (Petersen et al. 2007), i.e. all larval instar levels occur within the female abdomen. Adult flies can switch between host individuals but will never venture off-host. Only female bat flies will temporarily leave their hosts to deposit a single prepupa on the bat roost wall, the only developmental stage of a bat fly spent off host (Marshall 1970). After emergence from the pupa the imago uses carbon dioxide, as well as body heat and odour to locate a bat host (Lourenço and Palmeirim 2008b). It is unknown whether the same cues are used to discriminate between hosts.

Nycteribia kolenatii is a relatively small bat fly with a length of $2-2.5 \mathrm{~mm}$ (Theodor 1967). It mainly parasitizes the Daubenton's bat Myotis daubentonii (Chiroptera: Vespertilionidae), a common Palearctic bat species which habitat spreads from western Europe up to Japan. Nycteribia kolenatii has been identified as a vector (Gardner et al. 1987) of Polychromophilus (Bioccala) murinus.

Polychromophilus spp. (Apicomplexa: Haemosporida) form a genus of malaria-like protozoan parasites that, though taxonomically classified under Haemoprotidae (Garnham 1966), are phylogenetically nested within the Plasmodium clade (Witsenburg et al. 2012). The dipteran part of the life cycle is similar to Plasmodium spp., with an oocyst developing on the gut wall (Mer and Goldblum 1947, Gardner and Molyneux 1988). The vertebrate stage differs, however, in that there is no erythrocytic merogony, and only the gametocytes (the form infectious to the dipteran vector) develop in the erythrocytes (Garnham 1966). Polychromophilus spp. infect insectivorous bats globally (Garnham 1973) and can reach high infection rates locally. In western Switzerland 51\% of the $M$. daubentonii population was found to be infected with P. murinus (Megali et al. 2011). Despite its large presence, little is known about the pathogenicity and virulence of the parasite for both vector and host (Corradetti 1936, Gardner et al. 1987).

\section{Sample collection}

Myotis daubentonii were captured during the seasons of 2010 and 2011 at dusk, using a harp trap positioned over the 'Sorge', a small wooded creek flowing through campus of the Univ. of Lausanne into the Lake of Geneva. While gravid and lactating females were immediately released upon capture, all other bats were used for sampling and ringed to prevent resampling. Each bat's forearm length (to the nearest $0.1 \mathrm{~mm}$ ) and mass (to the nearest $0.1 \mathrm{~g}$ ) were measured. As a measure of body condition we used the OLS residuals from a regression of body mass on forearm length (Schulte-Hostedde et al. 2005). Spinturnix andegavinus wing mites were counted by inspecting the wing membranes and the uropatagium. Finally all bat flies were collected from the fur, using soft forceps and by gently blowing carbon dioxide through the fur away from the bat's head. Bat fly species were identified by FW following Theodor (1967) and Aellen (1955).

Blood was obtained by puncturing the uropatagial vein with a $0.5 \mathrm{~mm}$ gauge needle. Between 5 and $30 \mu \mathrm{l}$ of blood were collected using microvettes with EDTA. From each blood sample, one drop of fresh blood was applied to a glass microscope slide to make smears. Slides were subsequently dried and immediately submerged in 100\% methanol for fixation. Finally, a 5\% Giemsa-stain was applied for one hour to stain the cells. The P. murinus parasites were identified by FW following Garnham (1966). Their abundances, from now on referred to as 'parasitemia', were estimated by scoring the number of gametocytes observed in each smear at $600 \times$ magnification for $15 \mathrm{~min}$.

After blood sampling, haemostatic cotton was applied to the punctured vein until bleeding ceased. If the bats were needed for the experiment described below, they were hand-fed with mealworms and released into an exterior aviary $(1.8 \times 1.15 \times 2.45 \mathrm{~m})$ equipped with roosting sites. Mealworms and water were provided ad libitum. Bats that were not needed for further experiments were released at the capture site on the same night. All bats were captured under the licenses no. 1317 and no. 1656, authorized by the Cantonal Veterinarian Service of Vaud, Switzerland. The collected bat flies were put in separate $2 \mathrm{ml}$ screw cap tubes punctured with air holes and equipped with moist cotton. To prolong their survival, the flies were kept at $4^{\circ} \mathrm{C}$ for the survival experiment or until usage in the host choice assessment the following day. 


\section{Host-choice assessment}

The host preference of the bat fly $N$. kolenatii was assessed as follows. Two bats, which previously had been freed of their bat flies and with known parasitemia, were placed in a bat cage $(50 \times 40 \times 30 \mathrm{~cm})$ with a small slit as a roost site and with mealworms and water provided ad libitum. Pairs were created based on their dissimilar parasitemia and matched by sex and age as much as possible. At 09:00, two male and two female bat flies, which were all individually marked with UV-fluorescent dye, were placed on each bat. These flies originated from other bats caught the same night. Every two hours (except at 19:00), both bats were scanned with a UV light to examine the presence and location of the marked bat flies. At 21:00, when the bats would become active, the final position of each fly was noted and the bats released. In total, 35 of these trials were performed, all with different bat flies and bats. Experimentation was performed under the license no. 2322, authorized by the Cantonal Veterinarian Service of Vaud, Switzerland.

To test for any preference of the bat flies, we used generalised linear mixed modelling with final host choice as the response variable and a binomial error distribution. As predictors we included the sex of the bat fly as well as the bat on which is started. All other predictors were expressed as the difference between the two potential hosts: parasitemia, forearm length, body condition and the number of bat flies originally collected from this bat at capture. The bat-pair identity was included as a random factor. All parameters were standardized to central mean and half standard deviation (Grueber et al. 2011). The dredge() function from the MuMIn package created the full set of possible submodels and the Akaike information criterion for small samples (AICc) was calculated for each. Model averaging was performed over the top modelset with $\triangle \mathrm{AICc}<4$ (Burnham and Anderson 2002) for natural averages, unconditional standard errors and confidence intervals. Shrinkage averages and relative importance were calculated based on the entire model set.

Next, we tested if any bat fly preference based on host parasitemia co-determined the natural distribution of bat flies among bats in the wild. We used generalized linear models with a negative binomial error distribution to model natural bat fly abundance (i.e. the total number of bat flies per bat) as a function of host parasitemia, sex, age, number of Spinturnix andegavinus mites (a potentially competing ectoparasite), body condition, forearm length, year, date, and the interactions between year and date, year and parasitemia, age and condition and sex and condition. A graphical inspection of 'date' (expressed as number of days since the first of April) suggested a possible quadratic relationship with the number of bat flies, and therefore a quadratic term was also included. Parameter standardization, model set creation, model selection and multimodel inference were performed as described above for the host choice experiment.

\section{Bat fly survival}

In June 2010 and 2012, all flies were removed from ten wild-caught $M$. daubentonii and kept in separate tubes as described above, without food but with moist cotton. The off-host survival of these flies was monitored after which each was tested for parasite infection. To prolong their survival and thus gain resolution, the flies were kept at $4^{\circ} \mathrm{C}$ (Gardner and Molyneux 1988). Each day at noon, the bat flies were moved to ambient temperature and examined for signs of life (e.g. movement of legs, pumping abdomen). Motionless bat flies were coaxed from inactivity by flicking their tubes. If this still elicited no response, the fly was gently prodded with a blunt needle. Unresponsive flies were deemed dead and either stored in $70 \%$ ethanol $(2010)$ or frozen at $-80^{\circ} \mathrm{C}$ (2012) until further analysis.

Like all Hippoboscoidea, Nycteribiidae have their salivary glands in the abdomen, alongside the for- and midgut (Gardner and Molyneux 1988). Attempts at isolating the salivary glands proved unsuccessful. Instead, P. murinus infection was detected by DNA extraction and amplification. First, flies stored in alcohol were soaked in Millipore water for $2 \mathrm{~h}$. All flies were then triturated with sterile pestles. For the DNA extraction and purification, the Biosprint 96 tissue protocol was followed, with an overnight digestion. Eleven blank samples were included during the extraction process to check for possible contamination. The detection of $P$. murinus infection was done by amplifying a $705 \mathrm{bp}$ cytochrome b (cytb) fragment of the parasite following a nested PCR protocol. Primers, reagents and PCR temperature profile can be found in Megali et al. (2011). Bands, ran on a $1 \%$ agarose gel and stained with ethidium bromide, were visualized under UV light. Each sample was tested in duplicate. Samples that gave ambiguous results were retested. Any sample that remained unclear was removed from further analysis.

To confirm that the $P$. murinus-positive bat flies were actively infected (i.e. with oocysts or sporozoites), and exclude the possibility that we were only detecting an infected blood meal in the gut, each sample was tested for presence of $M$. daubentonii mtDNA. A $195 \mathrm{bp}$ fragment of the cyt $b$ gene was amplified using a nested PCR protocol (methods in Supplementary material Appendix 1). The inner primer pair was designed to specifically amplify $M$. daubentonii DNA and no dipteran nor human material. Again, each sample was tested in duplicate and any samples with conflicting results were reanalysed or excluded from the study.

To test for statistical difference in survival between infected and uninfected bat flies, a Cox proportional hazards regression model (CoxPHM) was applied to the survival data using the survival package for R. Instead of directly using the survival data, the CoxPHM uses the underlying hazard function. Comparing this function between groups allows the calculation of the hazard ratio $\left(b r_{A / B}\right)$, or the relative risk of group A compared to that of group B (Cox 1972). To deal with ties, Efron's approximation was used. We added year of the experiment (2010 or 2012) and the sex of the bat flies as covariates to the model, as well as the interactions between sex and infection status and year and infection status of the bat flies.

All statistical analyses were done in $\mathrm{R}$ ver. 2.15.0. All data of the host-choice experiment, the natural bat fly frequencies and the vector survival experiment are available at the Dryad repository (<http://datadryad.org/>) as doi:10.5061/dryad. $5 j 192$. 


\section{Results}

\section{Host choice assessment}

Over the 12 hour period that bats roosted together, a median of three bat fly exchanges (range $=0-7$ ) was observed, out of a potential 40 observable parasite exchanges per pair. The most common number of exchanges was two (Fig. 1a). Of the 280 bat flies, 177 were never observed switching hosts $(63.2 \%)$, the vast majority of those who did switch, switched once (Fig. 1b), and the average consecutive time spent on a specific host was $7.2 \mathrm{~h}$.

Due to the loss of some flies during the assays, the 35 host choice assays provided complete data for 198 bat flies, of which 110 were never observed to switch hosts (55.6\%). The top model of the logistic regression had only the starting position of the bat fly and relative parasitemia of the host as predictor variables and this pair of predictors starred in 16 out of the 17 top models, with the last model solely containing the starting position as a predictor (Supplementary material Appendix 2 Table A1). The bat on which a bat fly started the
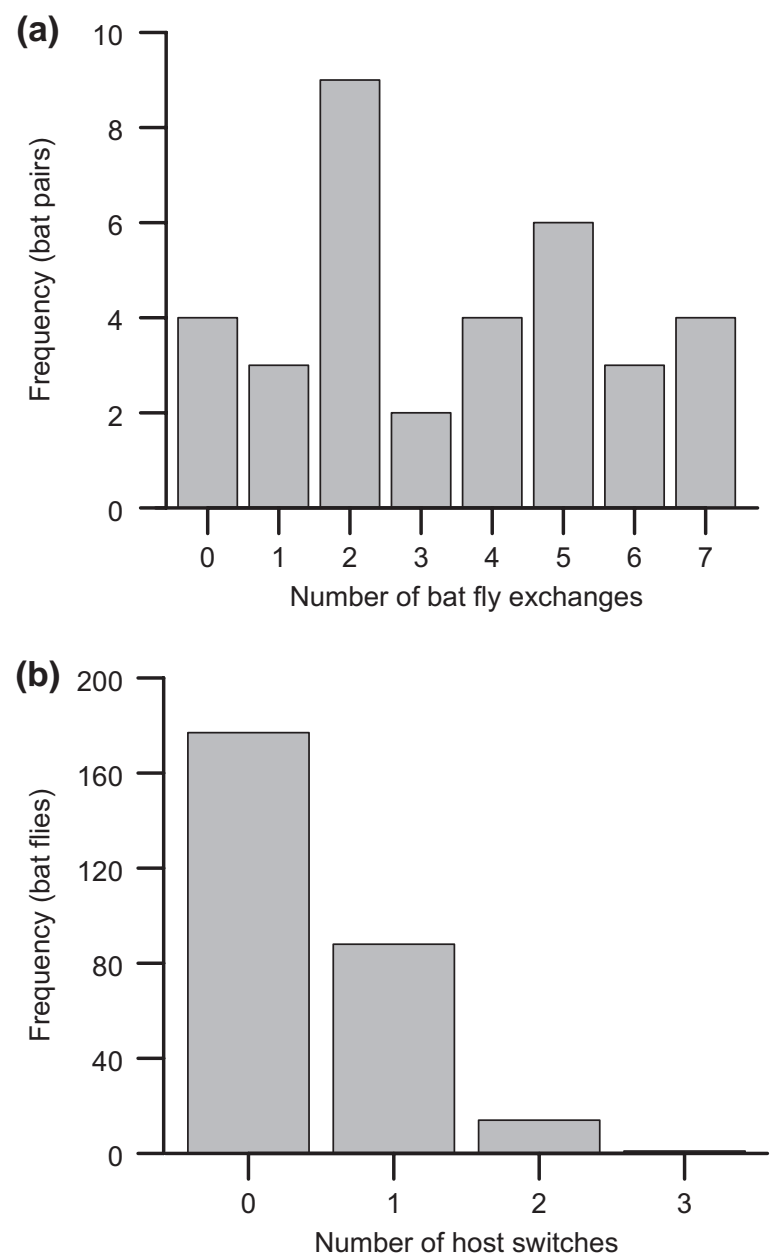

Figure 1. Bat fly host switching behavior. (a) From the host's perspective: the number of bat fly exchanges that occurred between the paired bats over the course of the 12-h experiment. (b) From the bat fly's perspective: the number of times a bat fly was observed to change hosts over the course of the 12-h experiment. A single individual was observed changing hosts three times. experiment had the highest importance, near unity. Second came relative parasitemia of the hosts, which had a negative influence on the bat fly's choice (Fig. 2a). Condition, the third most important parameter, was only half as important as parasitemia. Forearm length and original bat fly abundances were the least powerful predictors (Table 1).

\section{Natural bat fly abundances}

All 1116 bat flies collected from the 163 captured Myotis daubentonii were identified as Nycteribia kolenatii. Bat fly numbers per host ranged from 0-26 flies with a median abundance of 6 flies (mode $=2$, mean $\pm \mathrm{SD}=6.84 \pm 5.36$ ). The bat fly abundance data of the wild host population was described best by a model containing three terms: condition, length and date ${ }^{2}$; and these thee terms appeared consistently in all top models with the exception of one (Supplementary material Appendix 2 Table A2). Only condition and length had their estimate confidence interval not overlap with zero and both predictors showed a positive effect on bat fly abundances (Table 2, Supplementary material Appendix 2 Fig. A1). The third most important predictor date ${ }^{2}$ indicated that the number of bat flies a bat carried peaked in mid-August (Table 2, Supplementary material Appendix 2 Fig. A1). In $28 \%$ of bats gametocytes of Polychromophilus murinus were detected, yet these parasitemia had only marginal effect on the number of bat flies the host carried (Table 2, Fig. 2c).

\section{Bat fly survival}

A total of $177 N$. kolenatii bat flies were monitored in the survival experiment $(2010: \mathrm{n}=115 ; 2012: \mathrm{n}=62)$. In 13 individuals, the presence of malaria or host blood could not be unambiguously determined, and they were consequently excluded from any further analysis. The large majority $(92.7 \%)$ of bat flies tested positive for $M$. daubentonii mtDNA, including all the 27 flies that were positive for P. murinus $(16.4 \% ; 2010$ : males $=5$, females $=9 ; 2012$ : males $=9$, females $=4$ ). We decided to only analyse the individuals positive for $M$. daubentonii mtDNA to avoid any confounding effects. Nevertheless, repeating the analysis with all samples produced qualitatively the same results (data not shown). The sex of the bat flies had no effect on their survival $\left(\chi^{2}=0.0737, \mathrm{DF}=1, \mathrm{p}=0.786\right)$, nor its interaction with the infection status of the bat flies $\left(\chi^{2}=1.769\right.$, $\mathrm{DF}=1, \mathrm{p}=0.184)$. Bat flies carrying $P$. murinus survived significantly shorter, having an increased risk of dying $\left(h r_{\text {presentlabsent }}=1.74, \chi^{2}=7.084, \mathrm{DF}=1, \mathrm{p}=0.008\right.$; Fig. $3)$. The year of the experiment also had a clear effect on the survival of the bat flies, with bat flies in 2012 surviving longer and enduring a lower relative hazard $\left(h r_{2012 / 2010}=0.64\right.$, $\chi^{2}=5.689, \mathrm{DF}=1, \mathrm{p}=0.017$; Fig. 3). The effect of infection was independent on the year, as the interaction between year and infection status was not significant $\left(\chi^{2}=2.392\right.$, $\mathrm{DF}=1, \mathrm{p}=0.122$ ).

\section{Discussion}

In this study, we tested if the dipteran vector of the malaria-like parasite Polychromophilus murinus showed a 

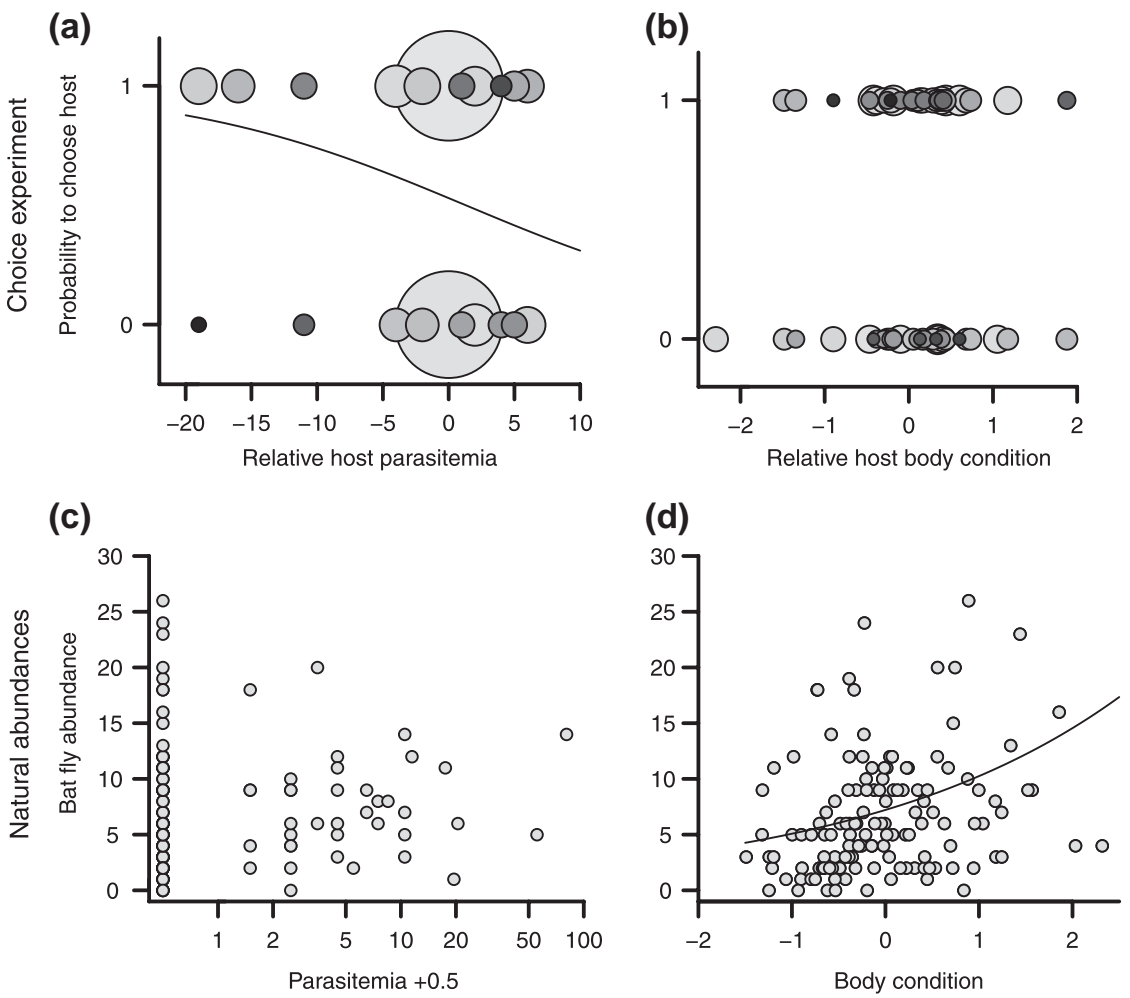

Figure 2. Comparing the results of the choice experiment (top) and natural bat fly abundances (bottom) based on host parasitemia (left) and host body condition (right). Note that the $\mathrm{x}$-axes in the top row represent the parameter relative to the other bat in the trial, whereas the $\mathrm{x}$-axes of the bottom row represent absolute values of single individuals. Top row: the probability of a bat fly to choose the focal host based on (a) the focal host's parasitemia level and (b) its condition, relative to that of the other bat present. The size and shade of the circles is relative to the number of bat flies for that combination of variables. Many bat flies had host-pairs with no observable difference in parasitemia, hence the large circles at 0 parasitemia. In these cases both hosts were chosen equally often as indicated by the same size of the circles. In contrast, when in a trial the focal bat host had much fewer parasites than the other available host (stongly negative parasitemia values), most bat flies chose the focal host (as indicated by larger circles at probability 1 ). This pattern proved much stronger for relative host parasitemia than for host body condition. Bottom row: bat fly abundance data from wild caught Daubenton's bats and (a) their parasitemia (log-scale +0.5$)$ and (b) their body condition. Body condition, but not host parasitemia proved a reliable predictor of vector abundances. Trendlines were produced according to best models.

feeding preference for hosts depending on their infection status. The behavioural assay demonstrated that the bat fly Nycteribia kolenatii has a preference for hosts that carry the least infective stages of the haemosporidian parasite, P. murinus. Moreover, we showed that bat flies infected with P. murinus have a decreased survival. However, we also demonstrate that this apparent feeding preference does not explain the vector's actual feeding pattern in the wild. Specifically, host parasitemia, the main host characteristic influencing the preference of $N$. kolenatii in the behavioural assays, had little predictive power over the natural feeding pattern of bat flies. Conversely, the two terms that could explain most variation in the natural distribution (host size and body condition) hardly influenced the vector's choice under controlled conditions.

\section{Choice experiment}

The majority of bat flies $(56 \%)$ were never observed to move between hosts, making the host on which a bat fly started

Table 1. Averages of model parameters of the host choice assays demonstrating that, apart from the starting position, the infection level of the host was the most important determinant of bat fly choice. Bat flies: the original number of bat flies found on the host upon capture. SE: standard error. $\mathrm{Cl}$ : confidence interval. The shrinkage average and importance were calculated over all models, the other statistics only over the top models with $\triangle \mathrm{AIC}_{\mathrm{C}}<4$.

\begin{tabular}{|c|c|c|c|c|c|c|}
\hline & $\begin{array}{l}\text { Natural } \\
\text { average }\end{array}$ & $\begin{array}{l}\text { Unconditional } \\
\text { SE }\end{array}$ & $\begin{array}{c}\text { Lower } \\
\mathrm{Cl}\end{array}$ & $\begin{array}{c}\text { Upper } \\
\mathrm{Cl}\end{array}$ & $\begin{array}{c}\text { Shrinkage } \\
\text { average }\end{array}$ & Importance \\
\hline Intercept & 0.20 & 0.17 & -0.14 & 0.54 & 0.20 & 1.00 \\
\hline Start bat & 1.07 & 0.32 & 0.45 & 1.69 & 1.06 & 0.99 \\
\hline Parasitemia & -0.97 & 0.41 & -1.77 & -0.17 & -0.86 & 0.89 \\
\hline Condition & 0.44 & 0.40 & -0.35 & 1.23 & 0.17 & 0.40 \\
\hline Length & 0.27 & 0.34 & -0.39 & 0.93 & 0.09 & 0.32 \\
\hline Bat flies & -0.33 & 0.43 & -1.16 & 0.51 & -0.09 & 0.32 \\
\hline
\end{tabular}


Table 2. Averages of model parameters of the natural distribution of the bat flies among their bat hosts, demonstrating that bat flies tended to reside on large hosts in good condition. SE: standard error. Cl: confidence interval. The shrinkage average and importance were calculated over all models, the other statistics only over the top models with $\triangle \mathrm{AlC}_{\mathrm{C}}<4$.

\begin{tabular}{|c|c|c|c|c|c|c|}
\hline & Natural average & Uncond. SE & Lower $\mathrm{Cl}$ & Upper Cl & Shrinking average & Importance \\
\hline Intercept & 1.98 & 0.10 & 1.78 & 2.17 & 1.97 & 1.00 \\
\hline Condition & 0.54 & 0.14 & 0.26 & 0.82 & 0.52 & 0.99 \\
\hline Length & 0.33 & 0.13 & 0.08 & 0.59 & 0.29 & 0.86 \\
\hline Date $^{2}$ & -0.68 & 0.47 & -1.61 & 0.25 & -0.58 & 0.82 \\
\hline Year & 0.18 & 0.22 & -0.25 & 0.61 & 0.07 & 0.49 \\
\hline Parasitemia & 0.17 & 0.13 & -0.08 & 0.41 & 0.08 & 0.47 \\
\hline Age & -0.01 & 0.14 & -0.30 & 0.27 & -0.01 & 0.37 \\
\hline Sex & -0.03 & 0.14 & -0.31 & 0.25 & -0.01 & 0.31 \\
\hline Date $^{2} \times$ Year & -1.68 & 0.86 & -3.37 & 0.02 & -0.50 & 0.28 \\
\hline Spinturnix sp. & 0.09 & 0.14 & -0.19 & 0.36 & 0.02 & 0.27 \\
\hline Date & -0.06 & 0.15 & -0.36 & 0.24 & 0.00 & 0.26 \\
\hline Age $\times$ Condition & 0.40 & 0.29 & -0.18 & 0.97 & 0.07 & 0.16 \\
\hline Sex $\times$ Condition & - & - & - & - & 0.00 & 0.07 \\
\hline Parasitemia $\times$ Year & - & - & - & - & 0.00 & 0.06 \\
\hline
\end{tabular}

the experiment the strongest predictor of host choice. If host parasitemia plays a role in bat fly host selection one would expect $50 \%$ of flies not to move, or at least end up at the host they started off, based on the experimental setup. This could partly explain the high rate of stationary bat flies. However, these flies presumably did not have complete information at their disposal and therefore might have made an 'uninformed' decision. A more likely explanation for the high rate of stationary flies is that bat flies might well be reluctant to switch hosts. Leaving the current host, irrespective of its quality, imposes a cost to the bat fly in the form of the risk of not finding another host, preferably more suitable than the current one. For a majority of bat flies, the risk of infection did not seem to outweigh the risks associated with leaving the current host.

Most $N$. kolenatii thus preferred to stay on their current host. However, the second most important factor influencing host choice was the host parasitemia. Of the $44 \%$ of bat flies that did move, the majority ended up on the host with the least parasites. This pattern of host preference was most

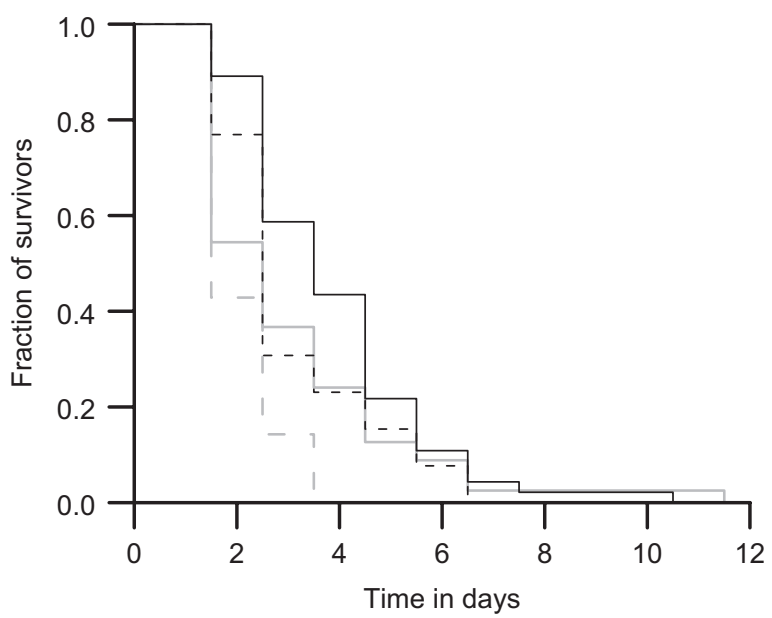

Figure 3. Survival of bat flies in relation to the presence of the parasite Polychromophilus murinus and the year of the experiment. Grey lines correspond to 2010, while black lines show data from 2012. Continuous lines indicate the absence of $P$. murinus, while dashed lines indicate the presence of $P$. murinus. obvious when the hosts had highly divergent levels of infection (Fig. 2a), indicating that one the two hosts was suffering from a severe infection. This could have increased the need of the bat flies to leave that particular host.

Parasites can cause their vectors to change their host preference behaviours, either as adaptive manipulation of the host by the parasite or an adaptive response of the vector to its infection (Cator et al. 2012). Irrespective of who is in charge, the fitness consequences of a certain host choice might very well be different for an infected vector compared to those of an uninfected vector. A shortcoming of our experimental setup was that, as we were not able to isolate the salivary glands, we could not asses the infection status of the bat flies unequivocally. We can therefore not tell if it were mainly the infected bat flies that preferred uninfected hosts, or, alternatively, that infected bat flies had lower levels of activity and therefore were those that never left their initial host.

After removing the bat flies from their original host, we were required to keep them at $4^{\circ} \mathrm{C}$, to prevent any major losses before the actual experiments. These temperatures are within the natural range of Myotis daubentonii and are encountered by $N$. kolenatii during the winter season (Gardner and Molyneux 1988). But even a cold shock can already alter insect behaviour (Chown and Terblanche 2007). Though this has never been formally assessed for bat flies, we should be cautious when interpreting our results. The cold shock might have caused a reduction in overall activity levels of the flies, reducing the number of host switches observed or caused changes in a combination of physiological traits which indirectly could influence decision making.

The experimental setup could not prevent the bats from grooming themselves. Although grooming has been hypothesized to be the main source of mortality for bat flies (Marshall 1970), its effectiveness has been questioned; ter Hofstede and Fenton (2005) found no relation between ectoparasite load and the amount of grooming performed by the bat host. Moreover, bats often seem oblivious to the presence of bat flies, showing no response to their biting (Dick and Patterson 2006) and several studies observed little to no response of bat flies to their hosts' grooming (Overal 1980, Fritz 1983).

Despite these shortcomings, we conclude that some of the bat flies in this experiment demonstrated a host 
preference for the least infected hosts. To our knowledge, this is the first study to indicate a preference of any member of the Hippoboscoidea based on the host's parasite status. Members of this superfamily are implicated in the transmission of a variety of endoparasites (e.g. Trypanosoma spp., Haemoproteus spp., Bartonella spp.; Baker 1967), including the Glossinidae-transmitted human sleeping sickness. An experiment performed on Trypanosoma congolense infected cattle could not demonstrate a clear preference of the vector Glossina pallidipes (Baylis and Nambiro 1993). Our finding that these bat flies may adjust their host choice according to the presence of (potentially dangerous) blood parasites warrants more in depth studies in other members of the Hippoboscoidea.

\section{Bat fly survival}

Independent of the mechanisms used by bat flies to select the least-infected hosts, the survival experiment demonstrated that evading $P$. murinus infections may be an adaptive behaviour. Though oocysts have been found in the local population of N. kolenatii (Supplementary material Appendix 2 Fig. A2), we were unable to demonstrate unambiguously any infection in the bat flies used for the survival experiment. Nevertheless, we found a negative effect of the presence of P. murinus on the survival of the bat flies, regardless of whether the parasites resided in the blood meal in the gut or were present in the form of oocysts or sporozoites. This reduction in survival could, therefore, be either a direct effect of the parasite on the fly's physiology, a more indirect effect, wherein the blood meal quality is lower in the presence of malaria, or other factors, unmeasured here, that influence mortality.

Negative effects of infection may often only surface when a vector suffers from other stressor at the same time (Lalubin et al. 2014). Our survival experiment did not only expose the bat flies to cold, but also to nutritional stress by keeping them off-host. Both stressors are expected to be less severe in the wild, but other sources of stress, such as competition and host grooming, may replace those. How this affects the parasite-induced mortality rates in natural populations, and therefore how strongly natural selection could act on any adaptive behaviours, is impossible to assess. Moreover, since nothing is known about the incubation period of $P$. murinus in $N$. kolenatii, it is hard to estimate the consequences of any increase in mortality for the transmission rate of the parasite.

Cost of infection to the dipteran vector is a matter of on-going debate (Ferguson and Read 2002). While the survival cost could be mitigated by a higher fecundity, the cost of infection could also act on the fecundity directly (Hurd et al. 1995). Female bat flies have, due to their viviparous life history, many more resources allocated to reproduction as compared to males. Consequently, an infection is expected to act differently on the two sexes. Though reproduction was not measured, the present study found no differences in survival between the sexes, suggesting that $P$. murinus directly acts on the survival of the bat flies. This is in contrast with a study concerning the viviparous hippoboscid fly Pseudolynchia canariensis, where females showed reduced survival when exposed to a haemosporidian parasite, but males did not (Waite et al. 2012).
The detection of $P$. murinus in the bat fly vector was low. Even if all the P. murinus PCR-positive bat flies were actually infected, the infection rate would only be $16 \%$. In contrast, we observed blood stages of the parasite in $28 \%$ of the hosts, and a previous study, based on PCR amplification of a cyt $b$ fragment of $P$. murinus, found an infection rate of $75 \%$ in the same population of $M$. daubentonii (Megali et al. 2011). The lower infection rate of the vectors compared to the host might be caused by an effective immune response of the bat fly, enabling them to quickly clear the infection. Moreover, the current study's choice experiment revealed some bat flies avoiding highly infectious hosts. If this behaviour is also shown by a subset of the wild $N$. kolenatii, it could lower the overall infection rate of this natural population. When an infection has the possibility to reduce the fitness of the vector, as suggested by this study, such avoidance behaviour would be the outcome expected of a coevolutionary arms race between a parasite and any of its hosts.

\section{Host switching behaviour}

Like most bats, $M$. daubentonii are highly social animals and will often huddle together when roosting. Based on the extremely close body contact of the bats, and the bat fly's capacity for rapid movements when agitated (Witsenburg unpubl.), the expectation was to see $N$. kolenatii move between the two hosts in the experiment continuously over the day. If flies moved continuously between hosts without distinction, the current location of any bat fly would have provided us with very little information on its main source of blood meals. Instead $63 \%$ of bat flies were never observed to leave the bat host on which they were released, and therefore fed on that host exclusively.

Though observations often readily describe the ease with which bat flies move between hosts (Marshall 1970, Fritz 1983), only one other study tried to quantify these host switches in a bat fly, and found that on average $52 \%$ of bat flies changed hosts after $24 \mathrm{~h}$ (Overal 1980) compared to $47 \%$ after $12 \mathrm{~h}$ in the current study. These rates seem similar enough to suggest that the bat fly mobility was not strongly affected by the cold shock prior to the experiment. Moreover, though these exchange rates may seem fairly low for such a mobile creature, from an epidemiological perspective these rates are extremely high, and any blood parasite transmitted by $N$. kolenatii should easily spread through the population. This in contrast to mosquitoes which blood feed only once before egg development. With a feeding interval of around 3-4 days, the speed of an infection outbreak is therefore expected to be lower by several orders of magnitude for mosquito transmitted haemosporidians.

Notably, the percentage of bat flies never leaving a particular host is probably a slight overestimate, since any bat flies that temporarily moved to the other host, but then returned before the subsequent observation, would not have been considered to have switched. Increasing the number of observations, however, would have meant disturbing the bats more often. Since Nycteribiidae are more prone to leave hosts which are stressed (Marshall 1971), more disturbances could also have artificially inflated the observed number of host switches. The current level of disturbance, handling and marking of bat flies may 
have already caused changes in their behaviour, which we cannot fully take into account.

\section{The natural distribution of bat flies}

The temperate zone bat fly populations show a peak in their population size in summer, which is normally synchronized to the birth of the young bats (Lourenço and Palmeirim 2008a). The bat flies, together with other ectoparasites, then migrate en masse to the new born (Christe et al. 2000, Lourenço and Palmeirim 2008a). Whether this is also true for $N$. kolenatii and its host $M$. daubentonii is not known, but this study found the highest bat fly densities on adults and subadults in August, well after the birth of the new young in June. However, since this was not the primary aim of this study, sampling did not occur regularly throughout the season. June samplings were indeed avoided so as not to disturb the bats during this period.

Parasitemia of the host was found to hardly have any effect on the $N$. kolenatii abundance of wild bats. Instead host body size and body condition were found to influence natural bat fly load most strongly. The positive relationship between bat fly numbers and body condition has been shown in a previous study and has been hypothesized to result from a preference of the bat flies (Reckardt and Kerth 2009). Choice experiments with Spinturnicidae wing mites and their bat hosts demonstrated a clear preference for well-fed individuals (Christe et al. 2003), whereas experiments with ornitophilic hippoboscid flies showed a preference for bird hosts in an average condition (Bize et al. 2008). In contrast, the choice experiment in the present study demonstrated no preference based on host condition.

The observed correlation between the body condition and bat fly abundance in the wild population might instead be explained by other factors than bat fly feeding preferences, e.g. an increased survival due to the higher nutritional value of well-fed hosts, a grooming-feeding tradeoff for the bat or social and/or spatial isolation of weaker individuals of the bat population. In general, many processes apart from preference determine an ectoparasite's - or any vector's - distribution. For example, the results of our behavioural assay indicate a certain reluctance of bat flies to exchange their currents hosts for another. If bat flies tend to stick with the host they first encounter, bat fly abundances should rather reflect host behaviour rather than that of the vector.

The link between vector behaviour and actual transmission of parasites in the field have still not been convincingly demonstrated (Cator et al. 2012). This study contributes to this discussion by demonstrating that the vector distribution cannot be inferred from the feeding preference of the vector, nor that an ectoparasite's natural distribution pattern is a reliable indicator of host attractiveness. These discrepancies may often simply be explained by the logistic constraints of an experiment. In our case, the decision to save vectors by keeping them at lower temperatures should prevent us from extrapolating our findings to the natural population. And indeed, we show here that these inferences do not work.

If preference cannot be translated into a relative increase of feeding on certain hosts, other methods will be required to estimate this important variable of epidemiological modelling (Kingsolver 1987). The increasing resolution of genetic typing already allows us to recognize not only host species, but known individual host blood donors from a vector's gut content (Cornet et al. 2013). The reducing costs of whole genome sequencing will soon make it feasible to read most of a host's heritable characteristics from the vectors blood meal. Yet other phenotypic traits such as the host's age and body condition would require different 'low-tech' methods, allowing the observation of natural biting behaviour without disturbance of vector and host. The Polychromophilus model system conveniently allowed for these observations since the vector is an ectoparasite. Such observations are valuable when many of the world epidemics are still vector transmitted.

Acknowledgements - This study has been supported by grants no. 31003A-120479 and 31003A-138187 by the Swiss National Science Foundation. We thank the Centre de Coordination Ouest pour l'Etude et la Protection des Chauves-souris in Geneva for logistic support. We would like to thank L. Dutoit, L. Clément and all others who helped out in the field. Our gratitude goes to E. Clark for reviewing the English in this manuscript.

\section{References}

Aellen, V. 1955. Etude d'une collection de Nycteribiidae et de Streblidae (Diptera Pupipara) de la région paléarctique occidentale, particulièrement de la Suisse. - Bull. Soc. Neuch. Sci. Nat. 78: 81-104.

Baker, J. R. 1967. A review of the role played by the Hippoboscidae (Diptera) as vectors of endoparasites. - J. Parasitol. 53: 412-418.

Baylis, M. and Nambiro, C. O. 1993. The effect of cattle infection by Trypanosoma congolense on the attraction, and feeding success, of the tsetse-fly Glossina pallidipes. - Parasitology 106: $357-361$.

Bize, P. et al. 2008. What makes a host profitable? Parasites balance host nutritive resources against immunity. - Am. Nat. 171: 107-118.

Burnham, K. P. and Anderson, D. R. 2002. Model selection and multimodel inference. - Springer.

Cator, L. J. et al. 2012. Do malaria parasites manipulate mosquitoes? - Trends Parasitol. 28: 466-470.

Chown, S. L. and Terblanche, J. S. 2007. Physiological diversity in insects: ecological and evolutionary contexts. - In: Simpson, S. J. (ed.), Advances in insect physiology, Vol. 33, pp. 50-152.

Christe, P. et al. 2000. Variation in intensity of a parasitic mite (Spinturnix myoti) in relation to the reproductive cycle and immunocompetence of its bat host (Myotis myotis). - Ecol. Lett. 3: 207-212.

Christe, P. et al. 2003. Differential species-specific ectoparasitic mite intensities in two intimately coexisting sibling bat species: resource-mediated host attractiveness or parasite specialization? - J. Anim. Ecol. 72: 866-872.

Cornet, S. et al. 2013. Malaria infection increases bird attractiveness to uninfected mosquitoes. - Ecol. Lett. 16: 323-329.

Corradetti, A. 1936. Alcuni protozoi parassiti di Nycteribiidae del genere Listropoda. - Ann. Igiene 46: 444-460.

Cox, D. R. 1972. Regression models and life-tables. - J. R. Stat. Soc. B 34: 187-220.

Day, J. F. et al. 1983. Feeding patterns of mosquitoes (Diptera: Culicidae) simultaneously exposed to malarious and healthy mice, including a method for separating blood meals from conspecific hosts. - J. Med. Entomol. 20: 120-127.

Dick, C. W. and Patterson, B. D. 2006. Bat flies: obligate ectoparasites of bats. - In: Morand, S. et al. (eds), Micromammals 
and macroparasites: from evolutionary ecology to management. Springer, pp. 179-194.

Farajollahi, A. et al. 2011. 'Bird biting' mosquitoes and human disease: a review of the role of Culex pipiens complex mosquitoes in epidemiology. - Infect. Genet. Evol. 11: 1577-1585.

Ferguson, H. M. and Read, A. F. 2002. Why is the effect of malaria parasites on mosquito survival still unresolved? - Trends Parasitol. 18: 256-261.

Ferguson, H. M. and Read, A. F. 2004. Mosquito appetite for blood is stimulated by Plasmodium chabaudi infections in themselves and their vertebrate hosts. - Malar. J. 3:12.

Freier, J. E. and Friedman, S. 1976. Effect of host infection with Plasmodium gallinaceum on the reproductive capacity of Aedes aegypti. - J. Invertebr. Pathol. 28: 161-166.

Fritz, G. N. 1983. Biology and ecology of bat flies (Diptera, Streblidae) on bats in the genus Carollia. - J. Med. Entomol. 20: $1-10$.

Gardner, R. A. and Molyneux, D. H. 1988. Polychromophilus murinus: a malarial parasite of bats: life-history and ultrastructural studies. - Parasitology 96: 591-605.

Gardner, R. A. et al. 1987. Studies on the prevalence of Hematozoa of British bats. - Mammal Rev. 17: 75-80.

Garnham, P. C. C. 1966. Malaria parasites and other Haemosporidia. - Blackwell.

Garnham, P. C. C. 1973. The zoogeography of Polychromophilus. - Ann. Parasitol. Hum. Comp. 48: 231-242.

Grueber, C. E. et al. 2011. Multimodel inference in ecology and evolution: challenges and solutions. - J. Evol. Biol. 24: 699-711.

Hurd, H. et al. 1995. Interactions between bloodfeeding, fecundity and infection in mosquitos. - Parasitol. Today 11: 411-416.

Kingsolver, J. G. 1987. Mosquito host choice and the epidemiology of malaria. - Am. Nat. 130: 811-827.

Knols, B. G. J. et al. 1995. Differential attractiveness of isolated humans to mosquitoes in Tanzania. - Trans. R. Soc. Trop. Med. Hygiene 89: 604-606.

Lacroix, R. et al. 2005. Malaria infection increases attractiveness of humans to mosquitoes. - PLoS Biol. 3: 1590-1593.

Lalubin, F. et al. 2012. Potential evidence of parasite avoidance in an avian malarial vector. - Anim. Behav. 84: 539-545.

Lalubin, F. et al. 2014. Natural malaria infection reduces starvation resistance of nutritionally stressed mosquitoes. - J. Anim. Ecol. 83: 850-857.

Liebman, K. A. et al. 2014. Determinants of heterogeneous blood feeding patterns by Aedes aegypti in Iquitos, Peru. - PloS Neglected Trop. Diseases 8(2): e2702.

Lourenço, S. and Palmeirim, J. M. 2008a. Which factors regulate the reproduction of ectoparasites of temperate-zone cavedwelling bats? - Parasitol. Res. 104: 127-134.

Lourenço, S. I. and Palmeirim, J. M. 2008b. How do ectoparasitic nycteribiids locate their bat hosts? - Parasitology 135: 1205-1213.

Supplementary material (available as Appendix oik.01785 at $<$ www.oikosjournal.org/readers/appendix $>$ ). Appendix $1-2$.

Data accessibility

Host-choice experiment results, vector survival experiment results and data on bat fly frequencies, parasitemia and biometrics of wild $M$. daubentonii are available at the Dryad repository (<http://datadryad.org/ $>$ ) as doi: 10.5061/dryad.5j192.
Lyimo, I. N. and Ferguson, H. M. 2009. Ecological and evolutionary determinants of host species choice in mosquito vectors. - Trends Parasitol. 25: 189-196.

Marshall, A. G. 1970. The life cycle of Basilia hispida Theodor 1967 (Diptera : Nycteribiidae) in Malaysia. - Parasitology 61: $1-18$.

Marshall, A. G. 1971. Ecology of Basilia hispida (Diptera: Nicteribiidae) in Malaysia. - J. Anim. Ecol. 40: 141-154.

Megali, A. et al. 2011. Disease in the dark: molecular characterization of Polychromophilus murinus in temperate zone bats revealed a worldwide distribution of this malaria-like disease. - Mol. Ecol. 20: 1039-1048.

Mer, G. G. and Goldblum, N. 1947. A haemosporidian of bats. - Nature 159: 444.

Overal, W. L. 1980. Host-relations of the batfly Megistopoda aranea (Diptera: Streblidae) in Panama. - Univ. Kansas Sci. Bull. 52: $1-20$.

Petersen, F. T. et al. 2007. The phylogeny and evolution of host choice in the Hippoboscoidea (Diptera) as reconstructed using four molecular markers. - Mol. Phylogenet. Evol. 45: 111-122.

Reckardt, K. and Kerth, G. 2009. Does the mode of transmission between hosts affect the host choice strategies of parasites? Implications from a field study on bat fly and wing mite infestation of Bechstein's bats. - Oikos 118: 183-190.

Schulte-Hostedde, A. I. et al. 2005. Restitution of mass-size residuals: validating body condition indices. - Ecology 86: 155-163.

Smith, D. L. et al. 2014. Recasting the theory of mosquito-borne pathogen transmission dynamics and control. - Trans. R. Soci. Trop. Med. Hygiene 108: 185-197.

ter Hofstede, H. M. and Fenton, M. B. 2005. Relationships between roost preferences, ectoparasite density, and grooming behaviour of neotropical bats. - J. Zool. 266: 333-340.

Theodor, O. 1967. An illustrated catalogue of the Rothschild collection of Nycteribiidae. - British Museum (Natural History).

Thomás, F. et al. 2005. Parasitic manipulation: where are we and where should we go? - Behav. Process. 68: 185-199.

Tomas, G. et al. 2008. Determinants of abundance and effects of blood-sucking flying insects in the nest of a hole-nesting bird. - Oecologia 156: 305-312.

Tripet, F. 2009. Ecological Immunology of mosquito-malaria interactions: of non-natural versus natural model systems and their inferences. - Parasitology 136: 1935-1942.

Waite, J. L. et al. 2012. Sex-specific effects of an avian malaria parasite on an insect vector: support for the resource limitation hypothesis. - Ecology 93: 2448-2455.

Witsenburg, F. et al. 2012. The evolutionary host switches of Polychromophilus: a multi-gene phylogeny of the bat malaria genus suggests a second invasion of mammals by a haemosporidian parasite. - Malar. J. 11. 